\title{
Ambulanter Benzodiazepinentzug bei Opiatabhängigen
}

\author{
Outpatient Benzodiazepine-Withdrawal of Opiate-Addicts
}

\section{Zusammenfassung}

Fragestellung: Es sollte untersucht werden, inwieweit im Behandlungsrahmen einer Allgemeinpraxis bei substituierten Opiatabhängigen ein ambulanter Entzug von Benzodiazepinen möglich ist. Methode: Mithilfe von Diazepam als Ersatzpräparat wurde eine langsame Abdosierung vorgenommen. Die Dauer der Abdosierung wurde individuell festgelegt. Ergebnisse: Bei 7 Patienten wurde ein Benzodiazepinentzug durchgeführt, 2 dieser Patienten erreichten einen benzodiazepinfreien Urin. Beide erfolgreichen Patienten wiesen eine Benzodiazepin-Konsumphase von unter einem halben Jahr auf. Schlussfolgerungen: Die Untersuchung zeigt, dass sich bei substituierten Opiatabhängigen ein ambulantes Abdosierungsprogramm von Benzodiazepinen mit akzeptablen Erfolgschancen in den Behandlungsrahmen einer Allgemeinpraxis integrieren lässt.

\section{Schliuisselwörter}

ambulanter Entzug · Benzodiazepine · Opiatabhängigkeit · Substitutionstherapie

\section{Abstract}

Purpose: The question under investigation is whether, in the setting of a general practice and within the framework of a maintenance program, an outpatient withdrawal of opiate addicts from benzodiazepines is possible. Methods: With diazepam as a substitute a slow reduction in dosage was undertaken. The duration of the reduction was individually fixed. Results: A benzodiazepine withdrawal program was carried out with 7 patients. 2 of them achieved urine free of benzodiazepines. Both successful patients had been taking benzodiazepine for less than half a year. Conclusions: The investigation shows that an outpatient reduction program for opiate addicts from benzodiazepines in a maintenance program can be integrated into the setting of a general practice. The chances of success can be regarded as acceptable.

\section{Key words}

outpatient withdrawel · benzodiazepines · opiate dependency · maintenance program

\section{Einleitung}

Der Benzodiazepinbeikonsum unter Opiatabhängigen ist ein weltweites Problem. In Niedersachsen gaben 41 \% der Opiatabhängigen vor Aufnahme der Substitution Beikonsum von Benzodiazepinen (BZD) an [1]. Aus Kanada wird berichtet, dass 62,5\% der unbehandelten Opiatabhängigen BZD konsumierten [2]. Unter laufender Substitutionstherapie verringern sich diese Zahlen, dennoch werden in Deutschland noch bei 21,5\% der mit Methadon behandelten
Opiatabhängigen BZD im Urin nachgewiesen [3]. 37\% der substituierten Opiatabhängigen in Australien hatten entsprechend ihrer Anamnese im letzten Monat BZD-Beikonsum [4].

Es finden sich mehrere Motive zum Benzodiazepinkonsum bei Opiatabhängigen [5]. Am Anfang steht oft der Ersatz fehlenden oder die Ergänzung gestreckten Heroins. Weiterhin ermöglicht der gleichzeitige Konsum von Heroin und BZD ein intensiveres Rauscherlebnis. Viele Heroinabhängige benutzen die BZD zur 
Selbstmedikation ihrer verschiedenen psychischen Störungen oder bei psychischen Belastungssituationen. Hinzu kommen die Eigentherapie von Schlafstörungen und die Vermeidung von Entzugserscheinungen bei BZD-Abhängigkeit. Die unerwünschten Folgen des Benzodiazepinkonsums sind vielfältig [6]. Es entsteht eine Abhängigkeit mit Entzugserscheinungen bei fehlendem Substrat. Augenfällig ist die Sedierung, Stürze können hinzutreten. Die BZD-Konsumenten sind kognitiv beeinträchtigt mit Gedächtnis- und Konzentrationsstörungen. In der Amnesie finden sich Fehlhandlungen. Besonders unter sehr hohen Dosierungen kommt es zu unerwarteten Verhaltensauffälligkeiten wie Aggressionen. $\mathrm{Zu}$ erwähnen sind auch Potenzstörungen, die durch gleichgerichtete Opiatwirkungen verstärkt werden. Schließlich wird der Drogentod als Folge des Mischkonsums von BZD und Opiaten diskutiert. In Bayern wurden bei $72 \%$ aller Drogentoten BZD nachgewiesen [7], wahrscheinlich besteht eine synergistische Wirkung von Opiaten und BZD. Sowohl Benzodiazepine als auch Opiate wirken atemdepressiv. Die traditionelle Behandlung der BZD-Abhängigkeit bei substituierten Opiatabhängigen ist in Deutschland der stationäre Teilentzug. Neben den hohen Kosten der stationären Therapie ist die Krankenhausbehandlung oft mit einer wochenlangen Wartezeit verbunden, so dass es sinnvoll erscheint, die Durchführbarkeit des ambulanten Entzuges zu untersuchen. Zu diesem Thema existieren zwei Studien. In Baltimore/USA erreichten 7 von 18 (39\%) BZD-abhängigen Methadonpatienten einen BZD-negativen Urin nach einer Entzugszeit von durchschnittlich 8 Wochen [8]. In Tel Aviv unterzog sich eine Gruppe von 33 Methadonpatienten einem ambulanten BZD-Entzug, 9 (27\%) waren nach 2 Monaten BZD-frei [9].

Die vorliegende Studie untersucht die Fragestellung, ob im Behandlungsrahmen einer Allgemeinpraxis ein ambulanter BZDEntzug durchführbar ist und mit welchen Erfolgschancen gerechnet werden kann.

\section{Methodik}

Sämtliche Substitutions- und Entzugsbehandlungen wurden in der eigenen Praxis vorgenommen, einer Allgemeinpraxis am Braunschweiger Stadtrand. Vor Start der Studie wurde im Rahmen einer gemeinsamen Aktion von drei Braunschweiger Substitutionspraxen für die Durchführung eines ambulanten BZD-Entzuges geworben. Die Patienten, die für den BZD-Entzug rekrutiert wurden, kamen entweder als neue Patienten aufgrund dieser Aktion in die Praxis oder es handelte sich um schon länger in der eigenen Praxis substituierte Patienten. Bei wiederholten ambulanten Entzügen wurde der erste in die Auswertung genommen.

Eine BZD-Abhängigkeit (Voraussetzung zur ambulanten Entzugstherapie) wurde angenommen, wenn im therapeutischen Bereich (max. $20 \mathrm{mg}$ Diazepam/Tag) mindestens 4 Monate, im Hochdosisbereich (mehr als $20 \mathrm{mg}$ Diazepam/Tag) mindestens 3 Wochen täglich BZD eingenommen wurden. Ziel des ambulanten Entzuges war das Erreichen der BZD-Freiheit, nachgewiesen durch Abgabe eines BZD-freien Urins.

Die standardisierte Anamnese umfasste u. a. Fragen zum Sozialstatus, zur psychischen Situation, zum Drogenkonsum und zur Motivation für die Einnahme von BZD. Beim Abschlussgespräch nach
Entzugsende wurde u. a. auf verstärkten Beigebrauch von anderen Suchtstoffen eingegangen.

Die Urinkontrollen wurden vor Beginn des Entzuges und während der Therapie einmal monatlich mit 7 Parametern durchgeführt (Opiate, Methadon, BZD, Barbiturate, Kokain, Amphetamine, THC), wöchentlich erfolgten Urinkontrollen mit 4 Parametern (Opiate, BZD, Kokain, THC). Als Substitutionsmittel wurde grundsätzlich Methadon verwandt, insofern wurde nicht auf Buprenorphin getestet. Die Analysen liefen als automatisierte Enzym-Immun-Assays (CEDIA) im Labor Dr. Kramer/Geesthacht. Vor Therapiebeginn wurde der Diazepam-Serumspiegel bestimmt zur Bestätigung der Einnahmemenge. Als therapeutischer Bereich wird 50-1000 ng/ $\mathrm{ml}$ angegeben, darüber liegt der Hochdosisbereich.

Der Entzug wurde durch allmähliches, semilogarithmisches Abdosieren des Ersatzpräparates Diazepam vorgenommen. Dieses wählten wir aufgrund der langen Wirkdauer aus. Die Apotheke lieferte das Medikament als 10-mg-, 5-mg- oder 2-mg-Tablette direkt an die Praxis. Der Patient erhielt weder Rezept noch Packung in die Hand. Die Anfangsdosis sollte der halben Dosis der letzten täglichen Einnahmemenge entsprechen. Diese so festgelegte Dosis stellte einen Richtwert dar, auf individuelle Wünsche der Patienten wurde eingegangen. Die gesamte BZD-Menge wurde entsprechend einer Äquivalenztabelle [6] auf Diazepam umgerechnet, dabei wurden $0,5 \mathrm{mg}$ Flunitrazepam $10 \mathrm{mg}$ Diazepam gleichgesetzt. Die Tagesdosis wurde in zwei Teile geteilt. Normalerweise erhielt der Patient 1/3 der Ration morgens in der Praxis, 2/3 als Mitgabe für den Abend. Der Mitgabeanteil wurde jedoch ggf. nach Wunsch des Patienten variiert. Die Wochenendration wurde komplett mitgegeben. Die Dosis wurde wöchentlich reduziert, dabei monatlich halbiert (semilogarithmisches Abdosieren). Auch beim Tempo des Abdosierens wurde in gewissem Maße auf die Wünsche des Patienten eingegangen. Die Behandlungsdauer richtete sich nach der Einnahmedauer von BZD. Bei mehrjähriger Konsumzeit wurde pro Jahr regelmäßigem BZD-Konsums ca. 1 Monat Therapiedauer eingeplant, bei kürzerer Konsumzeit ergab sich die Behandlungsdauer aus der Vorgabe des semilogarithmischen Abdosierens. Bei erheblichem zusätzlichem BZD-Konsum oder wiederholten Alkoholintoxikationen wurde die ambulante Therapie abgebrochen, ebenso galt die stationäre Einweisung zur Teilentgiftung von jeglichem Beikonsum für die Studie als Abbruchkriterium. Eine medikamentöse Krampfprophylaxe wurde nur vorgenommen bei Krampfanfällen mit Bewusstlosigkeit in der Anamnese. Bei Depressionen oder Ängsten erfolgte eine antidepressive Begleitmedikation, bei Schlafstörungen erhielten die Patienten kurzwirkende Neuroleptika oder sedierende Antidepressiva.

Vor Beginn des Entzuges wurde der Patient über den Behandlungsablauf und mögliche Entzugserscheinungen aufgeklärt. Er gab sein schriftliches Einverständnis.

\section{Ergebnisse}

Sieben Entzüge bei 7 Patienten aus dem Zeitraum zwischen dem 1.7.2001 und dem 30.6.2003 wurden in die Studie aufgenommen. Es handelte sich um 4 Frauen und 3 Männer, das Durchschnittsalter betrug 36 Jahre. Tab. 1 zeigt die sozialen Basisdaten sowie wesentliche psychische Symptome vor dem Entzug. 
Tab. 1 Soziale Daten und psychische Symptome vor dem Entzug

\begin{tabular}{llr}
\hline fester Wohnsitz & 7 & $100 \%$ \\
\hline Arbeitslosigkeit & 6 & $86 \%$ \\
\hline Hafterfahrung & 5 & $71 \%$ \\
\hline Depressivität & 2 & $29 \%$ \\
\hline Panikattacken & 5 & $71 \%$ \\
\hline Schlafstörungen & 5 & $71 \%$ \\
\hline
\end{tabular}

Die in Tab. 2 dargestellten Daten zur Suchtanamnese beziehen sich im Wesentlichen auf den BZD-Konsum.

Tab. 2 Suchtanamnese

\begin{tabular}{lc}
\hline Dauer Opiatabhängigkeit (Jahre) & $17(11-27)$ \\
\hline Dauer BZD-Konsum (Monate) & $81(1-240)$ \\
\hline $\begin{array}{l}\text { letzte Einnahmemenge } \\
\text { (mg Äquivalenzmenge Diazepam) }\end{array}$ & $141(20-280)$ \\
\hline Diazepamspiegel vor Entzug $(\mathrm{ng} / \mathrm{ml})$ & $1903(538-3400)^{1}$ \\
\hline
\end{tabular}

16 Patienten.

Die Diazepamspiegel „vor dem Entzug“ beziehen sich auf die letzte Zeit des Eigenkonsums, nicht auf die ersten Tage des Therapieprogramms. Alle Patienten konsumierten Diazepam, 3 allerdings zusätzlich Flunitrazepam mit Diazepam als Hauptdroge. Insofern wird mit dem Diazepamspiegel die durchschnittliche Gesamtbenzodiazepinmenge zu niedrig angegeben.

Es wurde von uns nach den Motiven für den BZD-Konsum gefragt. Die Antworten erfolgten als freie Formulierungen. Sie sind in Tab. 3 zusammengefasst, dabei konnte jeder Patient mehrere Motive nennen.

Tab. 3 Motive für BZD-Konsum, Mehrfachnennungen möglich

\begin{tabular}{|l|l|}
\hline Angst & 3 \\
\hline Schlafstörungen & 1 \\
\hline Erregungszustände & 1 \\
\hline Vergessen traumat. Erlebnisse & 1 \\
\hline Verstärkung Opiatrausch & 1 \\
\hline Gefühl der Stärke & 2 \\
\hline zum Leben dazugehörig & 1 \\
\hline
\end{tabular}

Eine Gruppe der Motive umfasst die Eigentherapie von psychischen Störungen, wobei die Angst ganz im Vordergrund steht. Darüber hinaus spielen die Verbesserung des Selbstwertgefühls und die Verstärkung des Opiatrausches eine Rolle.

Ein Behandlungserfolg wurde in 2 von 7 Therapien erzielt. Die übrigen 5 Therapien wurden abgebrochen. Eine Therapie von diesen 5 wurde wegen einer eingeschobenen stationären Opiatteilentgiftung als abgebrochen gewertet. Krampfanfälle traten während der Entzugsbehandlungen nicht auf. Im Abschlussgespräch wurden die
Patienten gefragt, ob sie während des Entzuges andere Drogen vermehrt konsumierten. Es ergaben sich folgende Antworten (Tab. 4, 5 Entzüge):

Im Folgenden werden charakteristische Entzugsdaten der 2 erfolgreichen Patienten aufgeführt (Tab. 5).

Tab. 4 Vermehrter Nebenkonsum während der Therapie

\begin{tabular}{lrr}
\hline Zigaretten & 2 & $40 \%$ \\
\hline Alkohol & 1 & $20 \%$ \\
\hline THC & 0 & $0 \%$ \\
\hline Heroin & 1 & $20 \%$ \\
Kokain & 0 & $0 \%$ \\
\hline
\end{tabular}

Beide Patienten zeichnen sich durch eine relativ kurze Konsumdauer von unter einem halben Jahr aus.

Tab. 5 Charakteristik der erfolgreichen Patienten

\begin{tabular}{|lrr|}
\hline & Patient 1 & Patient 2 \\
\hline Monate BZD-Konsum & 4 & 1 \\
\hline Konsummenge (Äquidosis Diazepam in mg) & 40 & 175 \\
\hline Diazepamspiegel (ng/ml) vor Entzug & 820 & 3400 \\
\hline Einstiegsdosis Entgiftung (mg Diazepam) & 40 & 150 \\
\hline Enddosis (mg Diazepam) & 1 & 2 \\
\hline Entzugsdauer (Wochen) & 24 & 23 \\
\hline UK auf BZD, 2 Mon. nach Entzug & neg. & neg. \\
\hline
\end{tabular}

\section{Diskussion}

Mit dieser Studie wird erstmals in Deutschland die Durchführbarkeit des ambulanten BZD-Entzuges bei Opiatabhängigen unter den Bedingungen der Substitutionstherapie untersucht. Bei unserem Kollektiv handelte es sich um eine kleine Gruppe Langzeitabhängiger (mittlere Dauer Opiatabhängigkeit 17 Jahre), deren Suchtkrankheit zu sozialen Folgeproblemen in Form von Arbeitslosigkeit und Hafterfahrung geführt hatte, die aber nicht durch Wohnungslosigkeit marginalisiert waren. Die durchschnittliche Dauer des BZD-Konsums war ebenfalls im Durchschnitt langjährig (knapp sieben Jahre), allerdings mit einer Spannbreite von einem Monat bis zu 20 Jahren. Als Motiv für den BZD-Konsum wurde ähnlich der Aufstellung von Tretter [5] die Selbstmedikation von psychischen Störungen genannt, dabei stand die Angst ganz im Vordergrund. Damit korrelierte die anamnestische Angabe von Panikattacken bei fünf unserer sieben Patienten. Hinzu trat als Motiv die Verstärkung des Opiatrausches, während der Heroinersatz bei den zumeist substituierten Patienten keine Rolle spielte. Darüber hinaus scheinen die BZD für einige Patienten eine Art „Supergefühl“ der Stärke zu vermitteln, das ebenfalls als Grund für den fortgesetzten Konsum genannt wird.

Die Entzugstherapien bei den sieben Patienten verliefen ohne schwere Komplikationen, insbesondere traten keine Krämpfe auf. Allerdings berichteten einige Patienten über vermehrten 
Konsum von Zigaretten, Alkohol und Heroin. Zwei Patienten mussten während der Therapie wegen Mischintoxikation bzw. zur Teilentgiftung von Opiaten stationär eingewiesen werden, die Therapie wurde daraufhin als Abbruch gewertet. Der Abbruchgrund der übrigen drei Patienten lag in fortgesetztem Eigenkonsum von BZD.

Zwei der sieben Patienten (29\%) beendeten den ambulanten Entzug erfolgreich. Damit war unsere Erfolgsrate fast identisch mit der einer israelischen Arbeitsgruppe (27\%) [9]. In einer amerikanischen Studie konnte eine höhere Erfolgsrate von 39\% erreicht werden [8], allerdings wurden nur Patienten mit einer Äquivalenzdosis von unter $6 \mathrm{mg}$ Alprazolam (entspricht $40 \mathrm{mg}$ Diazepam) zur ambulanten Entzugstherapie zugelassen. Die Erfolgsrate einer stationären Teilentgiftung von BZD-Beikonsum bei substituierten Patienten ist selbstverständlich höher. Specka et al. berichten, dass 79\% der stationären Patienten am Ende des Entzugs einen BZDfreien Urin aufweisen [10]. Allerdings werden viele der stationär Entzogenen nach der Entlassung rasch wieder rückfällig. Im gleichen Kollektiv reduziert sich der Anteil erfolgreicher Patienten drei Monate nach der Entlassung auf 13\%. Die Autoren kommen zu dem Schluss, dass ambulante Entzugsverfahren in die Substitutionstherapie eingeführt werden sollten, um die Erfolgsrate nach dem Entzug zu erhöhen. Bei unseren beiden erfolgreichen Patienten ließen sich zwei Monate nach Abschluss der Entzugsphase keine BZD im Urin nachweisen. Die Erfolgsrate des ambulanten Entzuges ist also zwar im Vergleich zur stationären kurzfristig deutlich geringer, es ergibt sich möglicherweise aber ein Vorteil bei längerfristiger Beurteilung. Ein Grund dafür könnte in der längeren Behandlungszeit der ambulanten Therapien liegen. Während der stationäre Entzug in dem Kollektiv von Specka auf durchschnittlich 16 Tage beschränkt ist [10], dauerten die Therapiekurse in den beiden ambulanten Studien sechs Wochen [9] bzw. durchschnittlich acht Wochen bei individueller Behandlungsdauer [8]. Die Abdosierungszeiten unserer erfolgreichen Patienten lagen sogar bei einem knappen halben Jahr. Die sehr langen Therapiezeiten ergaben sich als Folge der relativ hohen Anfangsdosierungen. Diese wiederum entsprachen nicht unserer Vorgabe der Hälfte der letzten Einnahmemenge, sondern wurden nach individuellen Wünschen der Patienten nach oben korrigiert.

Bei einem Vergleich der Ergebnisse zwischen stationärem und ambulantem Entzug muss erwogen werden, ob es sich bei den ambulanten Patienten um eine vorselektierte Gruppe handelt. Solch eine Vorauswahl fand jedoch in unserer Praxis nicht statt. In dem Untersuchungszeitraum wurden alle BZD-abhängigen Substitutionspatienten in das ambulante Entzugsprogramm aufgenommen. Eine stationäre Einweisung kam erst infrage nach dem Abbruch des ambulanten Entzuges.

Die beiden erfolgreichen Patienten zeichneten sich durch relativ kurze BZD-Konsumphasen von unter einem halben Jahr aus. Nicht ausgeschlossen sind allerdings frühere Konsumepisoden, die anamnestisch nicht erfasst wurden. Möglicherweise sind solche Patienten mit eher kurzer letzter Konsumdauer unabhängig von der Konsumhöhe besonders für die ambulante BZD-Entgiftung geeignet.

Wir benutzten als Austauschpräparat aufgrund der langen Wirkdauer Diazepam. Wie Weizman et al. [9] zeigen konnten, lässt sich die ambulante BZD-Entgiftung auch mit Clonazepam durchführen. Dieses mittellang wirkende Präparat hätte den Vorteil, dass sich über die Urinkontrollen oder Serumanalysen der Eigenkonsum von Diazepam aus der Drogenszene besser nachweisen ließe.

Insgesamt konnten wir zeigen, dass sich ein ambulantes Abdosierungsprogramm für BZD mit akzeptablen Erfolgschancen in das Setting der Substitutionstherapie integrieren lässt. Es sind jedoch weitere Studien mit größeren Patientenzahlen erforderlich, um Vor- und Nachteile verschiedener Entgiftungspräparate zu klären, genauere Empfehlungen für Dosierungen und Behandlungszeiten zu geben und möglicherweise besonders geeignete Patientengruppen zu definieren.

\section{Danksagung}

Der Dank geht an Prof. Dr. W. Poser für die Mithilfe bei der Vorbereitung der Studie.

\section{Literatur}

${ }^{1}$ Schulzke M. Methadon-gestützte Psycho-/Sozialtherapie für Heroinabhängige. Berichte zur Suchtkrankenhilfe. Niedersächsisches Sozialministerium, 1997

${ }^{2}$ Fischer B, Gliksman L, Rehm J et al. Comparing opiate users in methadone treatment with untreated opiate users: results of a follow up study with a Toronto opiate user cohort. Canadian Journal of Public Health 1999; 90: 299-303

${ }^{3}$ Wittchen HU. COBRA: Substitutionstherapie in der Routineversorgung. „Der Beikonsum von nicht verschriebenen Substanzen im 12-Monats-Verlauf“. Newsletter 2005; 10

${ }^{4}$ Darke S, Heather M, Hall W et al. Estimating drug consumption in opioid users: reliability and validity of a „recent use“ episodes method. British Journal of Addiction 1991; 86: $1311-1316$

5 Tretter F. Methadonsubstitution: Zur Problematik der zusätzlichen Verordnung von Benzodiazepinen. Diskussionspapier der BAS München, 2001

${ }^{6}$ Poser W, Poser S. Medikamente - Mißbrauch und Abhängigkeit Stuttgart, New York: Thieme, 1996

${ }^{7}$ Schmidt-Kittler H, von Meyer L. Drogenbedingte Todesfälle 1998. Suchtmedizin 2000; 2: $103-109$

${ }^{8}$ McDuff DR, Schwartz RP, Tommasello A et al. Outpatient benzodiazepine detoxification procedure for methadone patients. Journal of Substance Abuse Treatment 1993; 10: 297-302

${ }^{9}$ Weizman T, Gelkopf M, Melamed Yet al. Treatment of benzodiazepine dependence in methadone maintenance treatment patients: a comparison of two therapeutic modalities and the role of psychiatric comorbidity. Australian and New Zealand Journal of Psychiatry 2003; 37: $458-463$

${ }^{10}$ Specka M, Scherbaum N. Ergebnisse der stationären Entzugsbehandlung von Benzodiazepinen bei Methadonsubstituierten. Sucht 2005; 51: $329-338$ 